\author{
Naim Deniz Ayaz ${ }^{1 \star}$, Muammer \\ Goncuoglu², Omer Cakmak ${ }^{2}$ and Irfan \\ Erol $^{2}$
}

${ }^{1}$ Department of Food Hygiene and Technology,

Faculty of Veterinary Medicine, Kirıkkale University,

Krrkkkale, Turkey

${ }^{2}$ Department of Food Hygiene and Technology,

Faculty of Veterinary Medicine, Ankara University,

Ankara, Turkey

Dates: Received: 11 May, 2016; Accepted: 02 June, 2016; Published: 03 June, 2016

*Corresponding author: Naim Deniz

Ayaz, Department of Food Hygiene and

Technology, Faculty of Veterinary Medicine,

Kırıkkale University, Kırıkkale, Turkey, E-mail:

naimdenizayaz@hotmail.com

www.peertechz.com

Keywords: Campylobacter jejuni; hipO; ceuE; PCR

\author{
Research Article
}

\section{Comparison of hipO and ceuE Gene Based PCR Assays for the Detection of Campylobacter Jejuni}

\section{Introduction}

Campylobacter infections are one of the most prevalent zoonotic bacterial foodborne diseases of humans mostly caused by C. coli and C. jejuni. In the last decade, the prevalence of gastroenteritis caused by Campylobacter species were in an increasing trend [1]. In addition to enteritis, extraintestinal infections and sequelae may occur, including bacteremia, urinary tract infection, reactive arthritis and "GuillainBarre' syndrome" affecting the peripheral nervous system [2]. As C. jejuni has an ability to colonize and in some cases infect poultry intestine which makes poultry meat a significant reservoir and vehicle of foodborne campylobacteriosis [3].

In order to find out the prevalence of Campylobacter in poultry meat, routinely, conventional culturing technique is using in many food control laboratories [4]. Campylobacter species are known as fastidious microorganisms, so mostly it is hard to detect with conventional method and isolate by routine media [5]. In general, detection of Campylobacter species especially C. jejuni, is difficult and time consuming using conventional techniques. Therefore specific, sensitive and rapid methods are needed for the detection of Campylobacter spp. from food. To overcome these concerns many detection and molecular-based typing methods including PCR have been developed and used as an important and effective tool for the detection of Campylobacter spp. [6-10].

In order to detect $C$. jejuni from chicken feces, hippuricase (hipO) [11] and the enterochelin binding lipoprotein encoded by siderophore transport (ceuE) genes [12] were developed for PCR. In addition, specific PCR assays based on specific primer pairs were used to differentiate and identify C. coli and C. jejuni. In a study, standard isolation procedure and PCR assay was compared for the screening of Campylobacter in poultry. Results of this study showed that, PCR assay was clearly more sensitive and rapid than standard isolation procedure for the detection of the pathogen [5].
$\mathrm{N}$-benzoylglycine amidohydrolase (hippuricase) which is not present in C. coli, is an effective test to discriminate C. jejuni from C. coli phenotypically. Hippuricase activity is regulated by hipO gene [13] and can be detected by ninhydrin test, phenotypically [14]. Several tests which most of them are not standardized, are used in microbiology laboratories to find out the hippuricase activity [15]. After verifying that hipO gene is. only present in $C$. jejuni among Campylobacter species, gene of C. jejuni was cloned and sequenced to develop specific primers for the identification of C. jejuni [15]. Also, $c e u E$ gene which is an important virulence factor of Campylobacter spp and regulates siderophore transport system, specific primer pairs were developed for the detection both of the C. coli and C. jejuni $[12,16]$.

Therefore, this study was aimed to compare the specifity and sensitivity of hipO and ceuE gene based primers for the detection of C. jejuni by PCR.

\section{Materials and Methods}

Campylobacter isolates: In the present study, a total of 44 Campylobacter isolates including 41 Campylobacter jejuni, two C. coli and one C. lari were tested for the comparison of ceuE and hipO gene based PCR assays for the detection of Campylobacter jejuni. The isolates were recovered from turkey meat samples using conventional culture technique in a previous study [17]. C. lari NCTC 11352, C. coli ATCC 43478 and C. jejuni ATCC 33291 reference strains were used for the verification of the isolates tested for PCR analysis.

PCR analysis: In the study, ceuE [12] and hipO [11], genes based PCR assays were compared for the detection of $C$. jejuni. Primer pairs used in the ceuE and hipO genes based PCR assays were, Jej 1: 5'CCT GCT ACG GTG AAA GTT TTG C-3', Jej 2: 5'-GAT CTT TTT GTT TTG TGC TGC-3' and Hip 400 F: 5'-GAA GAG GGT TTG GGT GGT-3', Hip 1134 R: 5'-AGC TAG CTT CGC ATA ATA ACT TG-3 (Integrated DNA Technologies, IDT, Leuven, Belgium), respectively. 


\section{DNA extraction}

Chelex-100 (Bio-Rad, Hercules, CA, USA) was used for the DNA extraction of the isolates. All isolates that stored at $-86^{\circ} \mathrm{C}$ were grown in Bolton broth (Oxoid CM983 with supplement SR208, Hampshire, $\mathrm{UK}$ ) and incubated at $42^{\circ} \mathrm{C}$ for $24 \mathrm{~h}$ under microaerophilic conditions (CampyGen, Gas Generating Kit, Oxoid). From enrichment's one ml of broth was centrifuged at $12.000 \times g$ for 3 minutes and then solid phase was transferred into Chelex $100(200 \mu \mathrm{l}$ of $6 \%)$ before the addition of proteinase $\mathrm{K}(2 \mu \mathrm{lof} 20 \mathrm{mg} / \mathrm{ml})$. Mixture was incubated for 40 minutes at $55^{\circ} \mathrm{C}$ in thermomixer (Eppendorf Thermomixer 5437). The suspensions were heated in a boiling water bath for 8 minutes and then centrifuged at $12.000 \times g$ for 3 minutes. These DNA extracts were used as a template in the PCR analysis.

\section{DNA amplification for ceuE gene based PCR assay}

In $c e u E$ gene based PCR assay $25 \mu \mathrm{l}$ of master mix (Promega, Madison USA) that contains, $5 \mu$ l DNA extract, $1 \times$ PCR Buffer, 0.2 $\mathrm{mmol} / \mathrm{L}$ of each dNTP, $1.5 \mathrm{mmol} / \mathrm{L} \mathrm{MgCl}_{2}, 2 \mathrm{U}$ Taq DNA polymerase, $1 \mu \mathrm{mol} / \mathrm{L}$ of each primers was used. The DNA amplification was performed in a thermocycler (Biometra Personal Cycler, Goettingen, Germany) according to the protocol previously reported [12].

\section{DNA amplification for hipO gene based PCR assay}

In hipO gene based PCR assay $25 \mu$ of master mix (Promega) that contains, $5 \mu \mathrm{l}$ DNA extract, $1 \times$ PCR Buffer, $0.2 \mathrm{mmol} / \mathrm{L}$ of each dNTP, $1.5 \mathrm{mmol} / \mathrm{L} \mathrm{MgCl}_{2}, 2 \mathrm{U}$ Taq DNA polymerase and $0.4 \mu \mathrm{mol} / \mathrm{L}$ of each primers was used. The DNA amplification was performed as reported previously [11].

\section{Electrophoresis}

Resultant PCR products of each amplification process were subjected to ethidium bromide stained $(0.1 \mu \mathrm{g} / \mathrm{ml})$ 1.5\% agarose gel at $100 \mathrm{~V}$ for $1 \mathrm{~h}$. Electrophoresis gels were visualized and documented (Syngene Ingenius, Cambridge, UK). The expected PCR amplified DNA fragment sizes for ceuE and hipO genes were 793 bp and 735 bp, respectively.

\section{Results}

In the present study, 41 C. jejuni, two C. coli and one C. lari isolates were analyzed for the presence of hipO and $c e u E$ genes by PCR. By hipO gene based PCR analysis in all 41 (100\%) C. jejuni isolates 735 bp DNA fragment were shown and verified as $C$. jejuni. By ceuE gene based PCR analysis, in only 18 (43.9\%) out of 41 isolates 793 bp DNA fragment were detected and can be identified as $C$. jejuni. Both assays did not show reaction with $C$. lari and C. coli. The results of PCR analysis were given in Table 1.

According to the results, hipO gene based PCR analysis showed more specify and sensitivity than $c e u E$ gene specific PCR assay for the detection of C. jejuni.

\section{Discussion and Conclusion}

It was reported that all C. jejuni strains harbor hipO gene. However thermotolerant Campylobacter species other than C. jejuni are not the carrier of this gene [13-15]. This specific character of $C$. jejuni is utilized in culture technique for discriminating C. jejuni from
Table 1. Comparison between the results with hipO and ceuE based PCR assays.

\begin{tabular}{|c|c|c|c|c|}
\hline \multirow{2}{*}{ No } & \multirow{2}{*}{ Bacterial isolates } & \multirow{2}{*}{ Isolates code } & \multicolumn{2}{|c|}{ Results of the PCR assay } \\
\hline & & & ceuE & hipo \\
\hline 1. & C. jejuni & ATCC 33291 & + & + \\
\hline 2. & C. jejuni & $100-1$ & - & + \\
\hline 3. & C. jejuni & $100-2$ & - & + \\
\hline 4. & C. jejuni & $106-2$ & + & + \\
\hline 5. & C. jejuni & $106-3$ & + & + \\
\hline 6. & C. jejuni & $110-1$ & + & + \\
\hline 7. & C. jejuni & $110-2$ & - & + \\
\hline 8. & C. jejuni & $110-3$ & - & + \\
\hline 9. & C. jejuni & $111-3$ & - & + \\
\hline 10. & C. jejuni & $112-3$ & - & + \\
\hline 11. & C. jejuni & $116-1$ & + & + \\
\hline 12. & C. jejuni & $116-2$ & + & + \\
\hline 13. & C. jejuni & $170-2$ & - & + \\
\hline 14. & C. jejuni & $170-3$ & - & + \\
\hline 15. & C. jejuni & $184-1$ & + & + \\
\hline 16. & C. jejuni & $184-2$ & - & + \\
\hline 17. & C. jejuni & $184-3$ & - & + \\
\hline 18. & C. jejuni & $205-1$ & - & + \\
\hline 19. & C. jejuni & $210-2$ & - & + \\
\hline 20. & C. jejuni & $210-3$ & - & + \\
\hline 21. & C. jejuni & $242-1$ & + & + \\
\hline 22. & C. jejuni & $242-2$ & + & + \\
\hline 23. & C. jejuni & $242-3$ & + & + \\
\hline 24. & C. jejuni & 293-2 & - & + \\
\hline 25. & C. jejuni & 293-3 & - & + \\
\hline 26. & C. jejuni & $307-1$ & + & + \\
\hline 27. & C. jejuni & $307-2$ & + & + \\
\hline 28. & C. jejuni & $307-3$ & + & + \\
\hline 29. & C. jejuni & $310-1$ & - & + \\
\hline 30. & C. jejuni & $310-2$ & - & + \\
\hline 31. & C. jejuni & $376-1$ & - & + \\
\hline 32. & C. jejuni & $417-2$ & - & + \\
\hline 33. & C. jejuni & $418-1$ & - & + \\
\hline 34. & C. jejuni & $418-2$ & - & + \\
\hline 35. & C. jejuni & $418-3$ & - & + \\
\hline 36. & C. jejuni & $600-1$ & - & + \\
\hline 37. & C. jejuni & $646-2$ & + & + \\
\hline 38. & C. jejuni & 648-1 & + & + \\
\hline 39. & C. jejuni & $648-2$ & + & + \\
\hline 40. & C. jejuni & $650-1$ & + & + \\
\hline 41. & C. jejuni & $650-3$ & + & + \\
\hline 42. & C. coli & $649-3$ & - & - \\
\hline 43. & C. coli & ATCC 43478 & - & - \\
\hline 44. & C. lari & NCTC 11352 & - & - \\
\hline
\end{tabular}

C. coli. Detection of the hipO gene which is protected in C. jejuni using PCR, reported as an effective tool to identify the pathogen and differentiate from the other Campylobacter species [15,18]. And also the strains that were analyzed as hippuricase activity negative, can differentiate from C. coli in order to detect the hipO gene in C. jejuni strains $[18,19]$.

Hani and Chan [13], reported that, although 17 C. coli strains, C. sputorum, C. upsaliensis, C. lari and Helicobacter pylori were negative, 
12 C. jejuni strains were found positive by hippuricase gene probe. Also they detected, hippuricase-negative $C$. jejuni strains which were verified by DNA-DNA hybridization with hippuricase probe used in the study. These findings indicated that it is possible to identify hippuricase negative C. jejuni strains as $C$. coli according to the phenotypic hippuricase activity test [13].

Bang et al. [20], were obtained similar results with the findings of our study. In the study, ceuE and cadF based PCR methods were used to find out the virulence factors of pig and cattle $C$. jejuni isolates and three $C$. jejuni isolates were reported as $c e u E$ negative by PCR assay.

In another study, conventional cultivation method results were verified by both ceuE and hipO based PCR methods. Although three colonies were isolated as C. coli and three as $C$. jejuni by cultivation method, all the isolates harbored both $c e u E$ and hipO genes. So in the study it is concluded that, ceuE based PCR method is able to differentiate C. coli and C. jejuni from feces of chicken [5].

In a study two different results were found with hipO and ceuE genes based PCR assay. The C. jejuni specific hipO gene was detected from 25 isolates, 10 of them were interestingly hippurate negative and 15 of them were positive. However C. jejuni specific ceuE gene was only detected from 17 isolates, five of them were hippurate negative and 12 of them were positive. In the study, 36 out of 50 hippurate negative isolates harbored C. coli specific ceuE gene. Similar to our results, in the study, three $C$. jejuni isolates were not identified correctly by ceuE gene based PCR assay [21].

It is concluded that, in the present study all $41 \mathrm{C}$. jejuni isolates harbored hipO but only $18 \mathrm{C}$. jejuni isolates showed positive reaction by ceuE gene based PCR assay [12]. As C. jejuni is one of the most important foodborne bacterial pathogen for human, laboratories have to detect this pathogen without giving false negative results. The results of this study showed that hipO gene based PCR assay was more reproducibly and specific then $c e u E$ gene specific PCR assay for the detection and also confirmation of C. jejuni isolates. Although $16 \mathrm{~S}$ rRNA method is the most commonly used for identification of the micro-organism, use of hipO gene-based PCR will add value to the identification of $C$. jejuni.

\section{References}

1. Anon (2010) The community summary report on trends and sources of zoonoses, zoonotic agents and food-borne outbreaks in the European Union in 2008. EFSA J 8: 1496.

2. Nachamkin I, Allos BM, Ho T (1998) Campylobacter species and GuillainBarre' syndrome. Clin Microbiol Rev 11: 555-567.

3. Miller WG, Mandrell RE (2005) Prevalence of Campylobacter in the food and water supply: incidence, outbreaks, isolation and detection. 101-163. In: J Ketley, ME Konkel (Eds), Campylobacter: Molecular and Celular Biology. Horizon Scientific Press, Norfolk, UK.

4. Wedderkopp A, Rattenborg E, Madsen M (2000) National surveillance of Campylobacter in broilers at slaughter in Denmark in 1998. Avian Dis 44: 993-999.
5. Bang DD, Pedersen K, Madsen M (2001) Development of a PCR assay suitable for Campylobacter spp. mass screening programs in broiler production. J Rap Met Aut Microbiol 9: 97-113.

6. Debruyne L, Samyn E, De Brandt E, Vandenberg O, Heyndrickx M, et al (2008) Comparative performance of different PCR assays for the identification of Campylobacter jejuni and Campylobacter coli. Res Microbiol 159: 88-93.

7. Katzav M, Isohanni P, Lund M, Hakkinen M, Lyhs U (2008) PCR assay for the detection of Campylobacter in marinated and non-marinated poultry products. Food Microbiol 25: 908-914

8. Moreno Y, Herńandez M, Ferrús MA, Alonso JL, Botella S, et al. (2001) Direct detection of thermotolerant Campylobacter in chicken products by PCR and in situ hybridization. Res Microbiol 152: 577-582.

9. Sallam KI (2007) Prevalence of Campylobacter in chicken and chicken byproducts retailed in Sapporo area, Hokkaido, Japan. Food Cont 18: 11131120.

10. Uyttendaele M, Schukkink R, Van Gemen B, J Debevere (1995) Detection of Campylobacter jejuni added to foods by using a combined selected enrichment and nucleic acid sequence-based amplification (NASBA). Appl Environ Microbiol 61: 1341-1347.

11. Linton D, Lawson AJ, Owen RJ, Stanley J (1997) PCR detection, identification to species level, and fingerprinting of Campylobacter jejuni and Campylobacter coli direct from diarrheic samples. J Clin Microbiol 35: 25682572.

12. Gonzalez I, Grant KA, Richardson PT, Park SF, Collins MD (1997) Specific identification of the enteropathogenic Campylobacter jejuni and Campylobacter coli by using a PCR test based on the ceuE gene encoding a putative virulence determinant. J Clin Microbiol 35: 759-763.

13. Hani EK, Chan VL (1995) Expression and characterization of Campylobacte jejuni benzoylglycine amidohydrolase (hippuricase) gene in Escherichia coli. J Bacteriol 177: 2396-2402.

14. Harvey SM (1980) Hippurate hydrolysis by Campylobacter fetus. J Clin Microbiol 11: 435-437.

15. Slater ER, Owen RJ (1997) Restriction fragment length polymorphism analysis shows that the hippuricase gene of Campylobacter jejuni is highly conserved. Lett Appl Microbiol 25: 274-278.

16. Park SF, Richardson PT (1995) Molecular characterization of a Campylobacter jejuni lipoprotein with homology to periplasmic siderophore- binding proteins. J Bacteriol 177: 2259-2264.

17. Cakmak O, Erol I (2012) Prevalence of thermophilic Campylobacter spp. in turkey meat and antibiotic resistance of C. jejuni isolates. J Food Safety 32: $452-458$.

18. Steinhauserova I, Ceskova J, Fojtikova K, Obrovska I (2001) Identification of thermophilic Campylobacter spp. by phenotypic and molecular methods. J Appl Microbiol 90: 470-475.

19. Casadémont I, Bizet C, Chevrier D, Guesdon JL (2000) Rapid detection of Campylobacter fetus by polymerase chain reaction combined with nonradioactive hybridization using an oligonucleotide covalently bound to microwells. Mol Cell Prob 14: 233-240.

20. Bang DD, Nielsen EM, Scheutz F, Pedersen K, Handberg K, et al. (2003) $\mathrm{PCR}$ detection of seven virulence and toxin genes of Campylobacter jejuni and Campylobacter coli isolates from Danish pigs and cattle and cytolethal distending toxin production of the isolates. J Appl Microbiol 94: 1003-1014.

21. Nakari UM, Koivumäki A, Siitonen A (2006) Species identification of human Campylobacter strains. 1681. In: Proceedings of the 16th European Congress of Clinical Microbiology and Infectious Diseases ECCMID. Nice, France. 